\title{
Study of Film Cooling Appliedon a Fixed Blade of a Sector HPT of the CFM56-7B Engine
}

\author{
D. Cherrared
}

\begin{abstract}
We consider in this work a numerical three-dimensional study of the interaction of two row of discrete jets in a wall with an incompressible flow for an injection rate $\mathbf{M}=\mathbf{0 . 5}$. This simulation is applied to the stator blade of the CFM56-7B engine and is performed using the computational fluid dynamics (CFD) simulation tool, with CFX5.7.1 software. This code use the finite volume method to solve the equations of Navier-Stokes and offer several turbulences models. In this study, the shear stress turbulence model (SST) is used.The velocity and temperature distributions are presented and discussed. The results obtained show some information. The two rows of holes on the leading edge is not sufficient to cool the blade because the injection faces the hot flow (crossflow), and is found on the stagnation line of the leading edge. The cooling is only localized at the leading edge for the first row and extends over a certain distance from the intrados to the second row. More, the results show the existence of two tourbillions contrarotatifs. These vortices transport the hot gas in the jet and thus degrade the protective wall.
\end{abstract}

Index Terms-Film cooling, heat transfer, numerical simulation, turbulent flows.

\section{INTRODUCTION}

IT is well known from thermodynamic analysis that the performance of a gas turbine is strongly influenced by the temperature at inlet to the turbine. Modern gas turbine engines are designed to operate at inlet temperatures of $1800-2200{ }^{\circ} \mathrm{K}$ which are far beyond the allowable metal temperatures. Thus, to maintain acceptable life and safety standards, the structural elements need to be protected against the severe thermal environment. This calls for an efficient system. On such cooling technique currently used for high temperature turbines, is film cooling. The present paper aims to contributein the development of a prediction method for the simulation and to know the cooling behaviour. The application of computational fluids dynamics to film cooling problem is assessed in this study. Results are presented in terms of temperature and velocity calculation in the flow field.

The interaction of cool air jets with hot cross-flow fields is an interesting topic of research and occurs in a variety of industrial applications: pollutant discharges, vertical takeoff and landing (VTOL) engineering and aerodynamics, jets into combustors, waste disposal from smoke stacks into the atmosphere, film cooling of turbine blades, etc. The search for better performances for air plane engines has led to higher gas temperatures in the first stages of modern turbines. In

Manuscript received June 3, 2014; revised September 16, 2014.

D. Cherrared is with the Mechanical Engineering School, USTHB University, Bab Ezzouar Algiers Algeria, Algeria (e-mail: cherrared_d@yahoo.fr.) these operating conditions, the turbine blades need to be cooled to ensure their protection and achieve the projected life expectancy.

Discrete jet film-cooling is one of the techniques used to protect the blades that are particularly thermally exposed (see Fig. 1). The best compromise between admissible metal temperature and aerodynamic efficiency becomes a major objective in cooled blade design. In this technique, cooler air is injected into high temperature boundary layer on the blade surface. Since the injected cooler air is bled directly from the compressor before it passes through the combustion chamber, it represents a loss in the total power output.
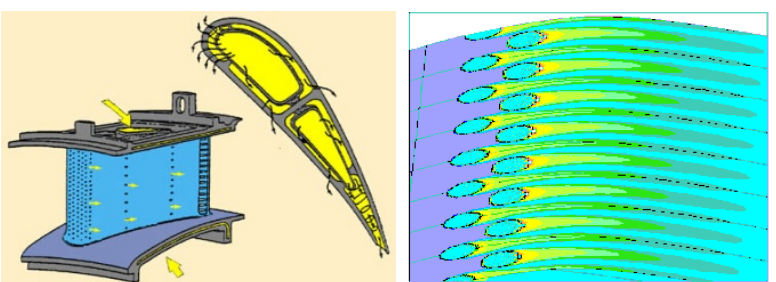

Fig. 1. General representation and visualization of film cooling.

A considerable effort has been devoted to understanding the coolant film behavior and its interaction with the mainstream flow (Chernobrovkin and Lakshminarayana [1], Hoda and Acharya [2]). The film cooling performance is influenced by the wall curvature, three dimensional external flow structures, free-stream turbulence, compressibility flow unsteadiness, the blowing rate ratio, the angle of injection and the geometry of the hole (Gartshore et al. [3]).

Analysis of the discrete-film-cooling performance requires an understanding of the fundamental jet-in-crossflow. The jet-in-crossflow problem has been investigated for over fifty years, and has been discussed by Goldstein [4] and Margason $[5]$.

CFD simulation is also an important method to study film cooling effectiveness and flow field of jet in crossflow in recent years. Xiao et al. [6] investigated the mixing characteristics of jet in cross flow using the CFD code Fluent. The three-dimensional mean and fluctuating characteristics of an impinging density jet in a confined cross flow were numerically investigated using the RNG turbulence model by Fan et al. [7].

The objective of this study is to elucidate the impact of the angular location of the discrete holes on the film-cooling effectiveness over the leading edge with two staggered rows of simple round holes, as determined by using the CFD technique. This part of the blade is directly exposed to the hot flow from the combustion chamber. The mainstream turbulence level fixed at $1 \%$. The density ratio is maintained at 1 but the tested blowing ratio is fixed to 0.5 .

The literature review reveals that RANS models are 
partially successful in simulating film cooling. The CFD modeling of film cooling holes can be achieved by various numerical methods of different complexity. In this study, the shear stress turbulence model (SST) is used.

The shear stress transport (SST) turbulence model offers significant advantages for non-equilibrium turbulent boundary layer flows and heat transfer predictions. ANSYS CFX complements the SST model with numerous other turbulence modeling innovations, including an automatic wall treatment for maximum accuracy in wall shear and heat transfer predictions and a number of extensions to capture effects like streamline curvature.

\section{PROBlem Description AND COMPUTATIONAL MODEL}

Fig. 2 shows the blade section to better visualize the injections holes configuration. We considered two rows of jets inclined at a compound angle and located on the leading edge of the blade.

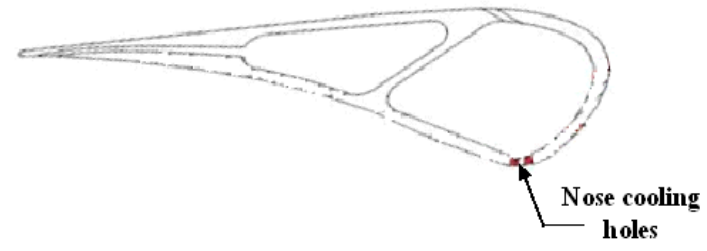

Fig. 2. Geometry of the blade.

The blade is $67 \mathrm{~mm}$ length and $20.8 \mathrm{~mm}$ in maximum width, it is provided with injection holes of diameter $D=0.6$ $\mathrm{mm}$. The geometry of the configuration injection holes studied here is shown in Table I.

\begin{tabular}{lllll}
\multicolumn{5}{c}{ TABLE I: GEOMETRIC PARAMETERS } \\
\hline \hline$\alpha$ & $\beta$ & $D[\mathrm{~mm}]$ & $X / D$ & $Z / D$ \\
\hline 85 & 35 & 0.6 & 3.3 & 3.8
\end{tabular}

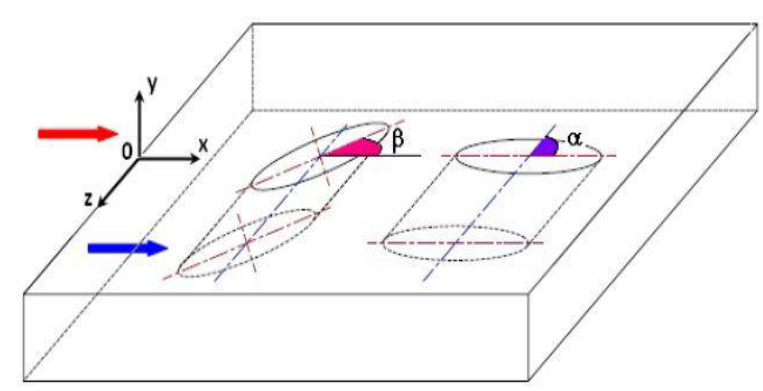

Fig. 3. The inclination angle $\alpha$ and the compound angle $\beta$ on a wall.

TABLE II: BOUNDARY CONDITIONS PARAMETERS

\begin{tabular}{|c|c|c|c|c|c|c|c|}
\hline \multirow{2}{*}{$M$} & \multicolumn{3}{|c|}{ crossflow } & \multicolumn{3}{|c|}{ Cooling jet } & \multirow{2}{*}{$\boldsymbol{\rho}_{\infty} / \boldsymbol{\rho}_{c}$} \\
\hline & $U_{\infty}(\mathrm{m} / \mathrm{s})$ & $T u_{\infty}$ & $T_{\infty}\left(K^{\circ}\right)$ & $U_{c}(\mathrm{~m} / \mathrm{s})$ & $T u_{c}$ & $T_{c}\left(K^{\circ}\right)$ & \\
\hline 0.5 & 100 & $1 \%$ & 1750 & 50 & $10 \%$ & 804 & 1 \\
\hline
\end{tabular}

In Table I, the distance $(Z / D)$ between injections holes is 3.8 times the diameter.

Fig. 3 visualizes the inclination angle $\alpha$ (located in the plane XOY) and the compound angle $\beta$ (located in the plane $\mathrm{XOZ}$ ).
The approach velocity of crossflow is about $100 \mathrm{~m} / \mathrm{s}$ with a temperature of $1750 \mathrm{~K}$ and a degree of turbulence of $1 \%$.

\section{NUMERICAL PROCEDURE}

To start the analysis, we specially pay attention in selected grid. The numerical domain was discretized using an unstructured tetrahedral grid illustrated in Fig. 4-Fig. 5.

Non uniform grid was generated and grid refinement close to the wall and injection hole zone was applied. Several successive grid refinements have been carried out in every case to get negligible effect of the mesh in the solutions.

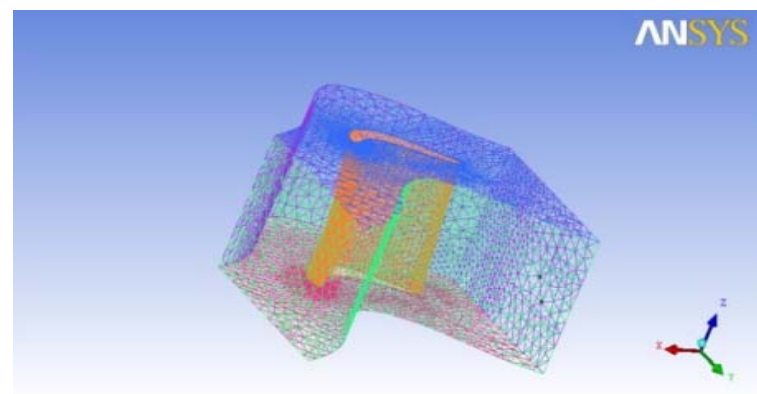

Fig. 4. Computational geometry.
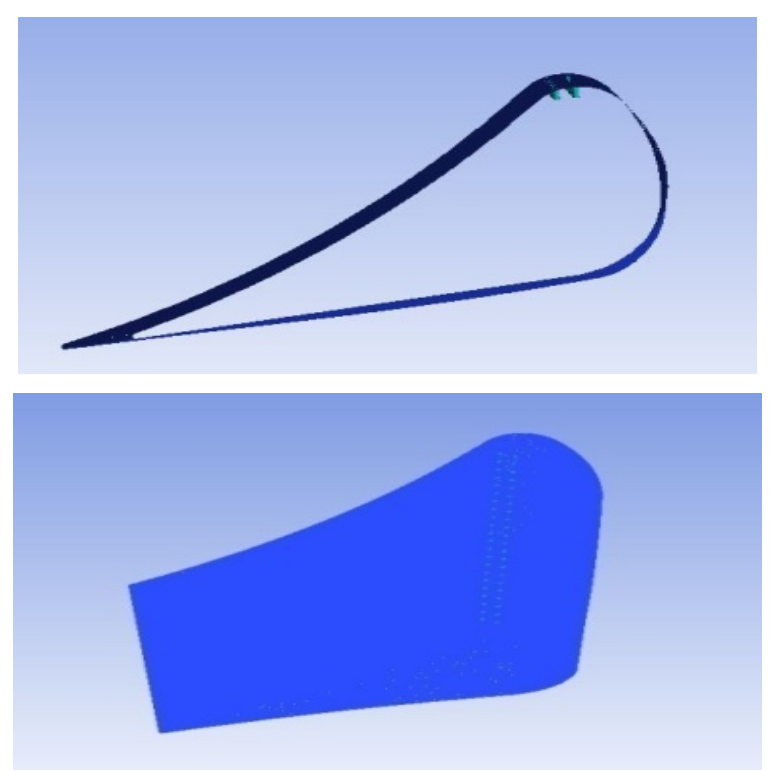

Fig. 5. Mesh of the holes

This study uses a commercial CFD code based on the finite volume method, CFX5. The flow is considered as incompressible and steady state and the turbulence fluctuations are governed by the Boussinesq eddy-viscosity assumption.

The SST turbulence model with automatic near wall treatment is used to predict the flow structure and heat transfer over the film-cooling surface. Automatic near wall treatment with automatically from wall functions to a low-Re near wall formulations as the mesh is refined. One of the well known deficiencies of the k- $\varepsilon$ model is its inability to handle low turbulent Reynolds number computations. Complex dumping functions can be added to the $\mathrm{k}-\varepsilon$ model, as well as the requirement of highly refined near wall grid resolution $\left(y^{+}\right.$ $<0.2$ ) in an attempt to model low turbulent Reynolds numbers flows. This approach often leads to numerical 
instability. Some of these difficulties may be avoided by using the $k-\omega$ model, making it more appropriate than the $\mathrm{k}-\varepsilon$ model for flows requiring high near wall resolution. However, a strict low Reynolds number implementation of the model would also require a near wall resolution of at least $y^{+}<2$. This condition cannot be guaranteed in most applications at all walls. For this reason, a new near wall treatment was developed by CFX for the k- $\omega$ based models that allows for a smooth shift from a low Reynolds number form to a wall function formulation. This near wall boundary condition, named automatic near wall treatment in CFX5, is used as the default in all models based on the $\omega$ equation (standard $\mathrm{k}-\omega$, SST ...ect.)

The three-dimensional incompressible Reynolds-averaged Navier-Stokes equations are solved by a second-order upwind scheme unrelated to the convective terms, whereas the energy terms are evaluated using a third-order QUICK scheme. The coupling between velocity and pressure in momentum equations is governed by the SIMPLE scheme.

The CFX solver finishes the calculations when the equation residuals calculated using the specified method is below the target residual value. A convergence criterion of $10^{-4}$ is used to ensure negligibly small iteration errors.

\section{RESUlts AND DiscussiOnS}

Fig. 6 represents the thermal distribution of the jets. They show the interaction between jet and crossflow. We notice that the jet has an almost periodic character for the holes of the first row and randomly for the holes of the second. This is due to the influence of the jets of the first row (interaction between the jets). The appearance of important contours confirms the existence of vortex structures resultant from the interaction between the main flow and the jet.

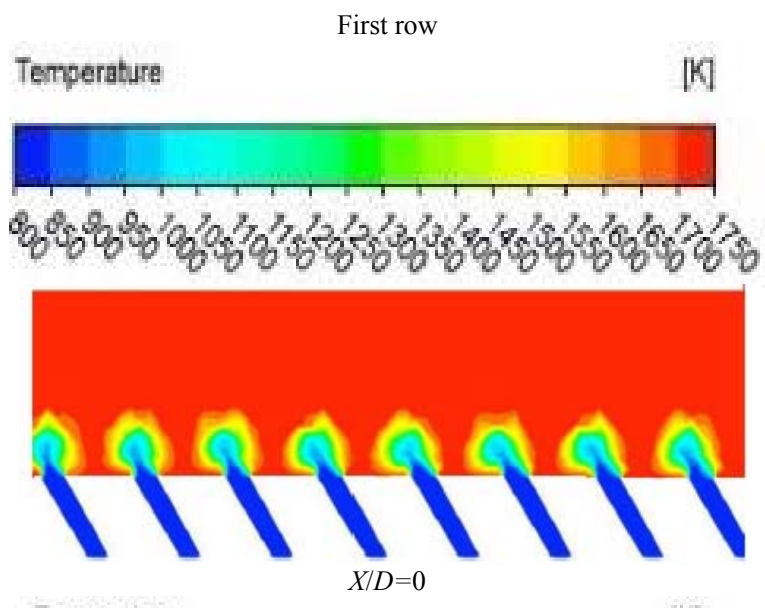

Temperature

(K)
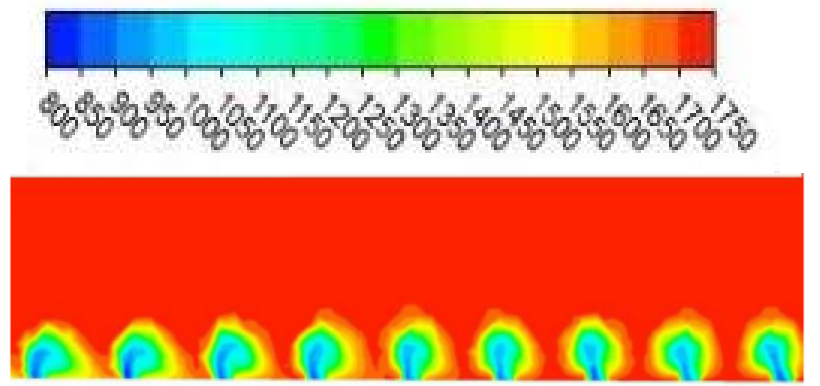

$X / D=1$
Temperature

[K]
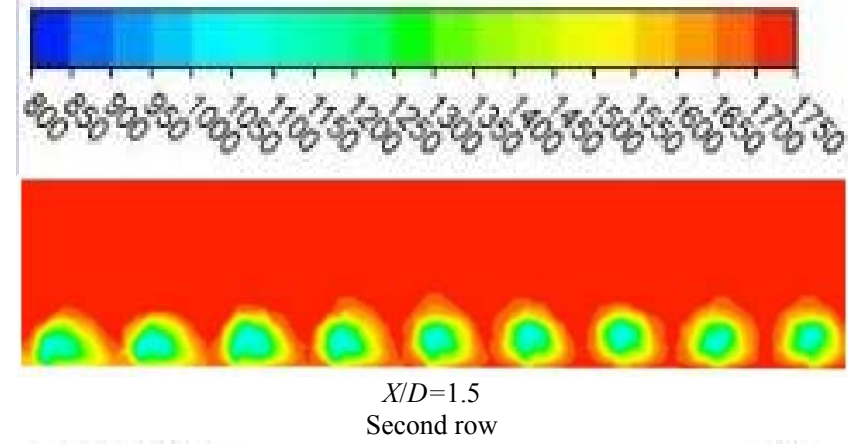

Temperature

[K]
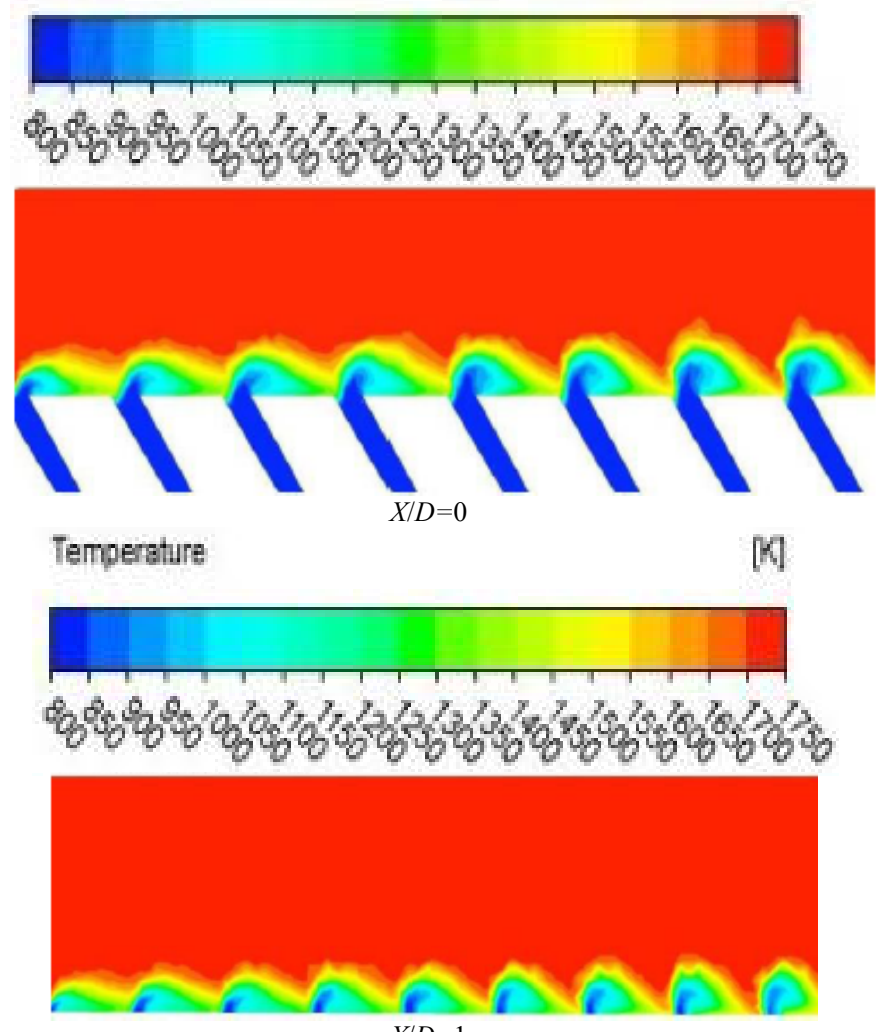

Temperature

[K]
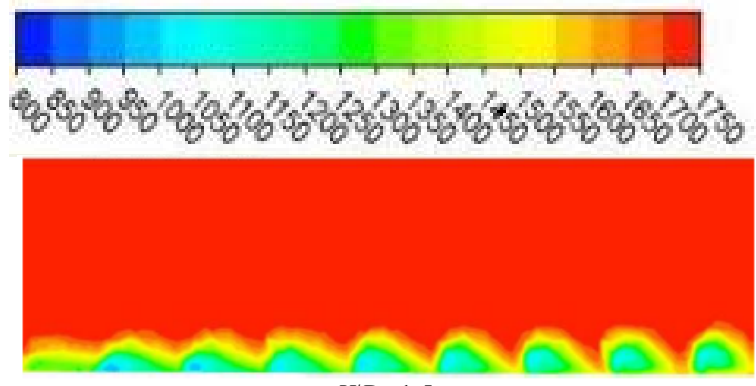

$X / D=1.5$

Fig. 6. Representation of the efficiency contours.

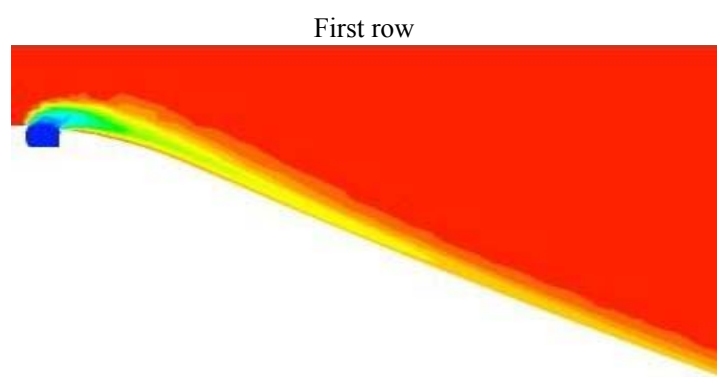




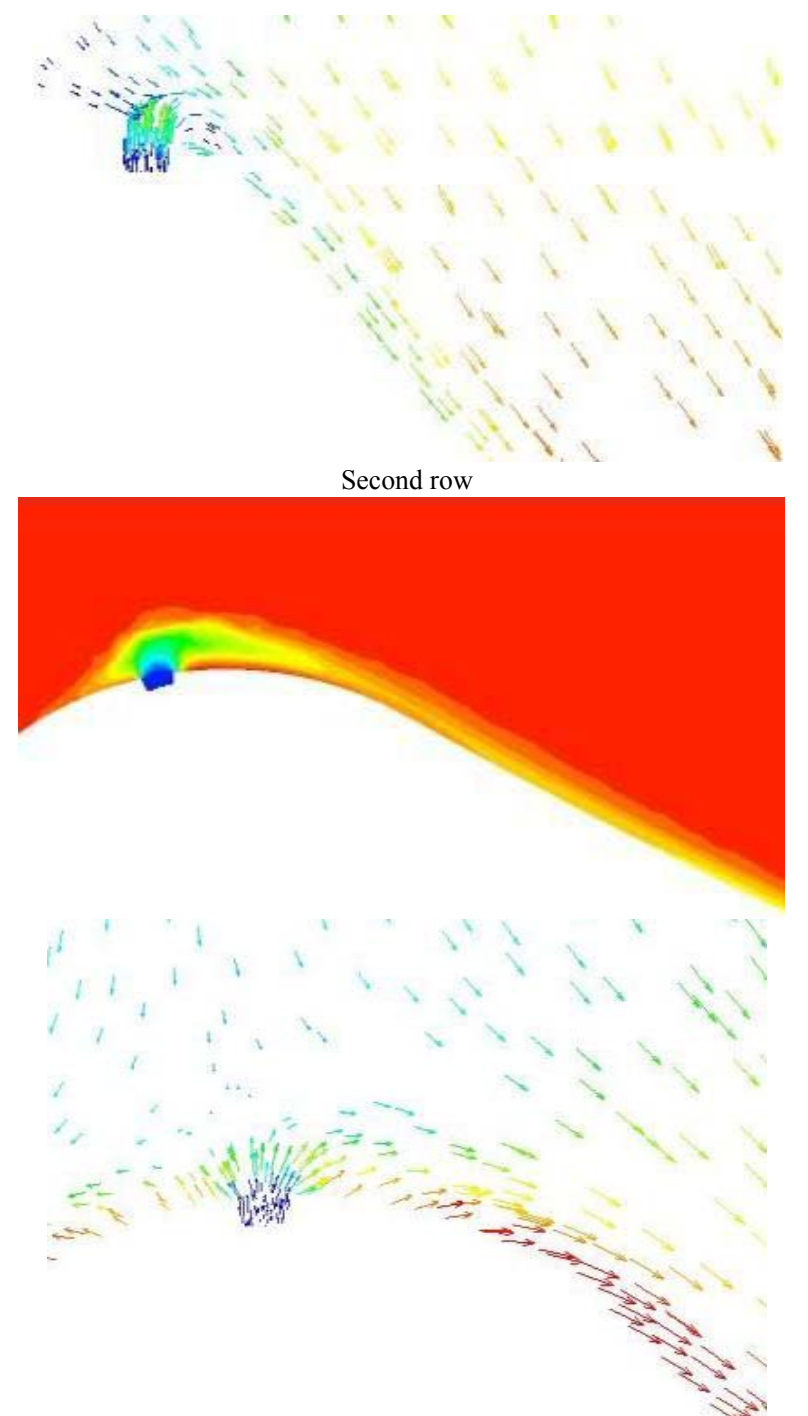

Fig. 7. The fields of velocity and temperature.
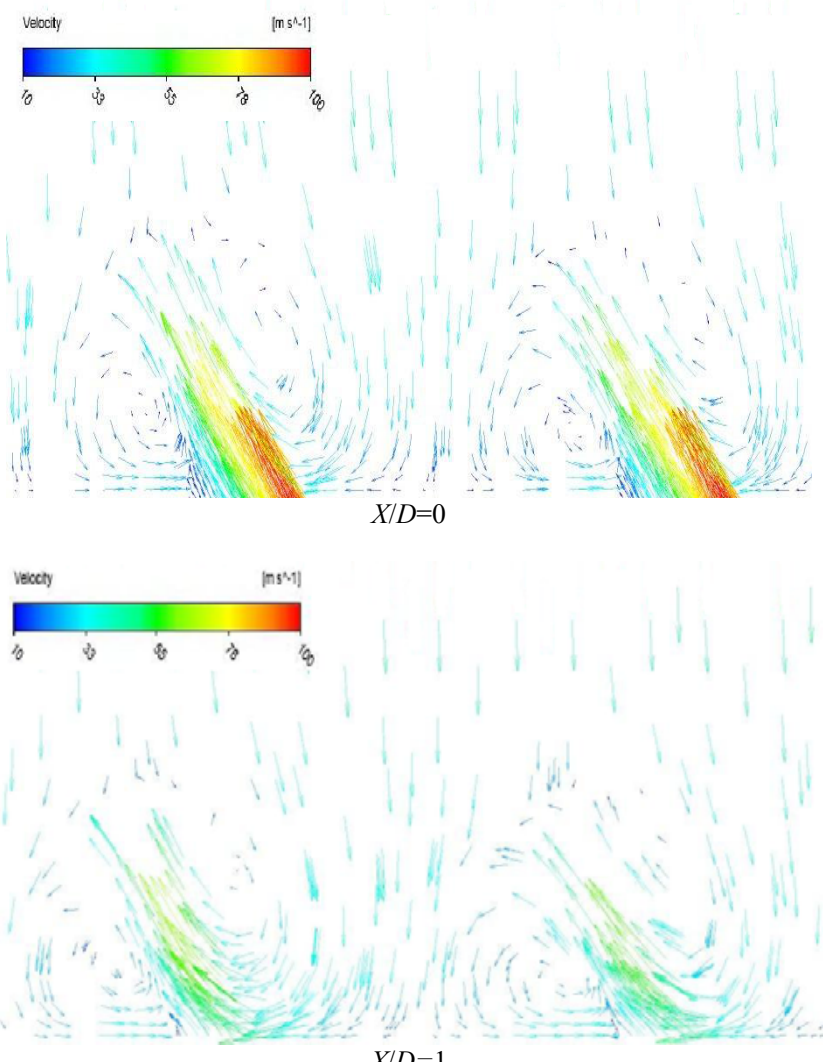

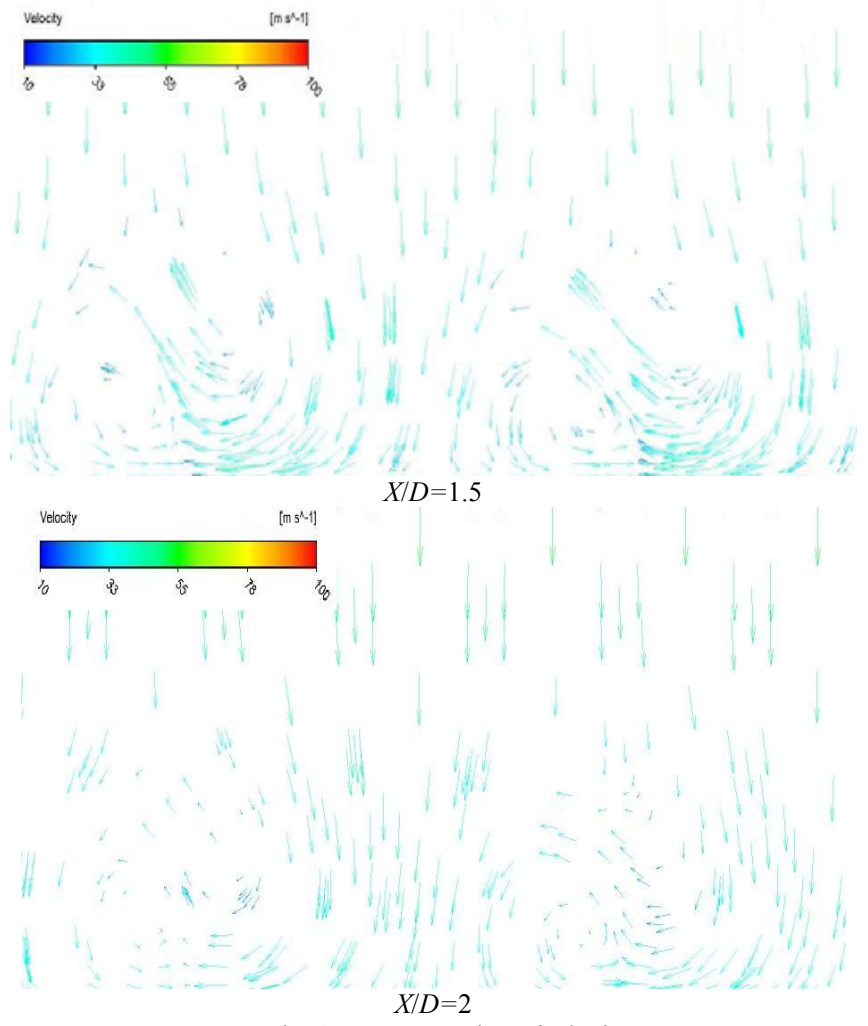

Fig. 8. Representation ofvelocity vectors.

Fig. 7 represents the velocity and temperature fields, on a plan passing by the injection hole. These figures show the interaction between the jet and the main flow. They illustrate the detachment of the jet and its attachment farther. We also notice that the main flow is dominant in this region and the jet is almost suffocated.

Fig. 8 represents vectors velocities on a perpendicular plan to different positions on the blade $(X / D=0, X / D=1, X / D=1.5$, $X / D=2)$ for a rate of injection $M=0.5$.

Vectors velocities represented in Fig. 8 show the existence of two vortex contrarotatifs and are more and more less intense by going away from the injection point (vortex loses its strength and decreases in size).

Fig. 9 shows the temperature distribution on the blade. It is noted that the cooling is only localized at the leading edge for the first row and extends over a certain distance from the intrados to the second row. This confirms that two rows of holes on the leading edge is not sufficient to cool the blade because the injection faces the hot flow (crossflow), and is found on the stagnation line of the leading edge.

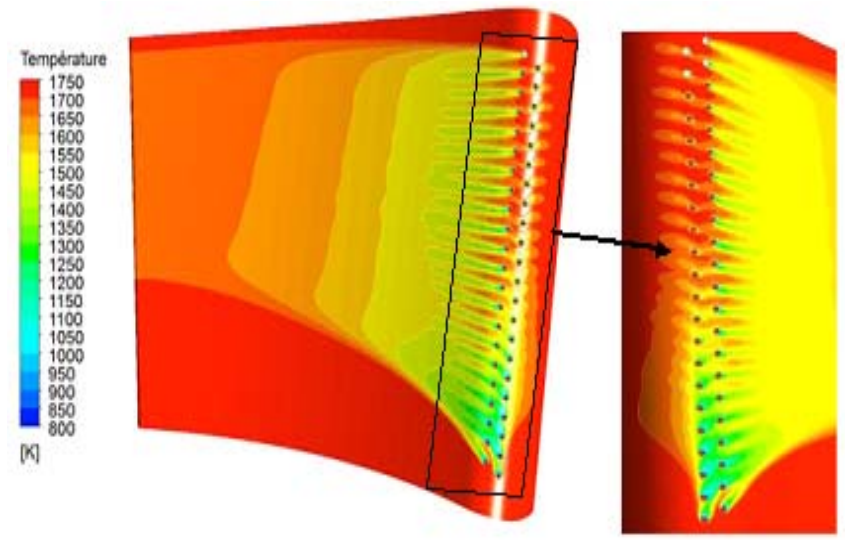

Fig. 9. The temperature distribution. 


\section{CONCLUSION}

The results obtained in this study showed that the structure of the jet is fairly complex; of three-dimensional nature and is strongly influenced by several parameters, both interaction between the hot gas flow and cooling jets or between themselves which are still subject to this complexity. The numerical simulations presented have shown the existence of two tourbillions contrarotatifs. These vortices transport the hot gas in the jet and thus degrade the protective wall.

\section{REFERENCE}

[1] A. Chernobrovkin and B. Lakshminarayana, "Numerical simulation and aerothermal physics of leading edge film cooling," Journal Power Energy, vol. 213, no. 2, pp.103-118, 1999

[2] A. Hoda and S. Acharya, "Predictions of a film coolant jet in cross-flow with different turbulence models," ASME Journal Turbomach, vol. 122, no. 3, pp. 558-569, 2000.

[3] I. Gartshore, M. Salcudean, and I. Hassan, "Film cooling injection hole geometry: Hole shape comparison for compound cooling orientation," American Institute of Aeronautics and Astronautics, vol. 39, no. 8, pp. 1493-1499, 2001.
[4] R. J. Goldstein, Advances in Heat Transfer, Academic Press, New York, 1971, pp. 321-379.

[5] R. J. Margason, Fifty Years of Jet in Cross-Flow Research, AGARDCP- 534, 1993.

[6] X. Yang and T. H. Wu," Numerical simulation of a horizontal momentum jet in cross-flow," Journal of Hydrodynamics, vol. 18, no. 1, pp. 118-125, 2006.

[7] F. J. Yu, W. D. Zeng, and Z. Yan, "Three-dimensional mean and turbulence characteristics of an impinging density jet in a confined crossflow in near field," Journal of Hydrodynamics, vol. 16, no. 6, pp. 737-742, 2004.

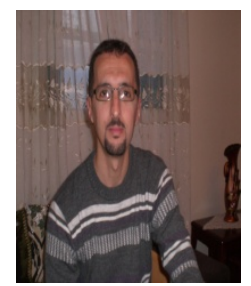

Djamel Cherrared was born on May 26, 1968 in Algeria. He received the master degree and the $\mathrm{PhD}$ degree in mechanical engineering in 1998 and 2009 from the USTHB University. Since 1999 he is a research professor in the Department of Mechanical Engineering at the USTHB University. $\mathrm{He}$ is a member of laboratory energy mechanics and conversion systems.

Djamel Cherrared is interested in research themes including fluid mechanics, turbulence, heat transfer, turbomachinery. 